\title{
Experimental Evaluation of a Data-Driven Control System using an Electronic Thermal Regulator
}

\author{
Yuka Okubo \\ Graduate School of Engineering, Hiroshima University, \\ 1-4-1, Kagamiyama, Higashihiroshima city, Hiroshima, Japan \\ Yoichiro Ashida \\ Graduate School of Engineering, Hiroshima University, \\ 1-4-1, Kagamiyama, Higashihiroshima city, Hiroshima, Japan \\ Takuya Kinoshita \\ Graduate School of Engineering, Hiroshima University, \\ 1-4-1, Kagamiyama, Higashihiroshima city, Hiroshima, Japan \\ Toru Yamamoto \\ Graduate School of Engineering, Hiroshima University, \\ 1-4-1, Kagamiyama, Higashihiroshima city, Hiroshima, Japan \\ E-mail: okubo-yuka@hiroshima-u.ac.jp, ashida-yoichiro@hiroshima-u.ac.jp,kinoshita--takuya@hiroshima-u.ac.jp, \\ yama@hiroshima-u.ac.jp \\ http://www.hiroshima-u.ac.jp
}

\begin{abstract}
IoT (Internet of Things) has been attracted attention, and several system data can be obtained by communication and it is stored in database. The input/output data is expected to be utilized to control systems in industries. The data-driven technique has been proposed as a way to utilize database for calculating PID gains. The scheme is effective for non-linear system control, but it is hard to be implemented to systems. It is due to restricted memory capacity. Therefore, a host computer calculates PID gains based on the data-driven scheme instead of a control device. The calculated gains are sent to a general controller from a computer. The effectiveness of the proposed platform is demonstrated by the experimental examination.
\end{abstract}

Keywords: PID controller, data-driven controller, electronic thermal regulator, FRIT

\section{Introduction}

In recent years, IoT has been received attention, and the data of systems obtained through a lot of sensors is hoped to be utilized to control systems in industries ${ }^{1}$. Such data, which is collected by a communication between sensors and cloud, is stored in database.

On the other hand, the electronic thermal regulators ${ }^{2}$, control devise, are employed to control systems, in which
PID controller ${ }^{3,4}$ is basically applied. In the case of using PID controller, the PID gains must be tuned considering the properties of controlled objects. Most of the practical controlled objects have nonlinearity, and it is hard to control them suitably by using the fixed PID controller. Therefore, the data-driven technique ${ }^{5}$ having effectiveness for non-linear systems has been proposed. Calculating a set of PID gains based on the database depending on current operating data, the data-driven scheme can appropriately control such systems. The data- 
driven technique needs the large memory for database. Therefore, it is hard to implement this technique to the electronic thermal regulator due to its restricted memory capacity. If the computer is utilized for implementing the data-driven control scheme, the new interface between the computer and controlled object must be introduced instead of the electronic thermal regulator. Hence, the data-driven scheme is hardly implemented to the industrial field.

In this paper, the construction of the data-driven control platform using the electronic thermal regulator is firstly explained, in which the database is introduced in order to realize the data-driven PID controller. According to the proposed platform, the host computer calculates PID gains by the data-driven technique and sends them to the control device. As a result, the aforementioned memory capacity restriction of the electronic thermal regulator can be avoided. Furthermore, the existing interfaces between electronic thermal regulator and control objects do not need to be changed.

In this paper, section 2 shows the schematic of the data-driven control platform. The design of a data-driven controller is explained in section 3 . In section 4 , the effectiveness of the proposed platform is verified by implementing it for an actual experimental equipment. The paper ends with concluding remarks in section 5 .

\section{Construction of the data-driven control platform}

The schematic of a data-driven control platform is shown in Fig. 1. In this paper, the electronic thermal regulator plays a role of a controller. The electronic thermal regulator receives control outputs, e.g. temperature, humidity or pressure through a sensor and determines the control input. In the proposed platform, the reference signal and control I/O data are sent to the host computer having a database from the electronic thermal regulator. The host computer sends a set of PID gains calculated on data-driven scheme to the electronic thermal regulator. Since the host computer sends PID gains instead of the control input, the electronic thermal regulator can control systems using last PID gains even if the communication is interrupted.

\section{Design of the data-driven controller}

\subsection{Controlled Object}

The controlled object can be described by the following nonlinear system:

$$
\text { ( ) } f . \quad(1)) \text {, }
$$

where ( ) is system output, $\cdot$ ) denotes the function of nonlinearity and -1 ) is an information vector

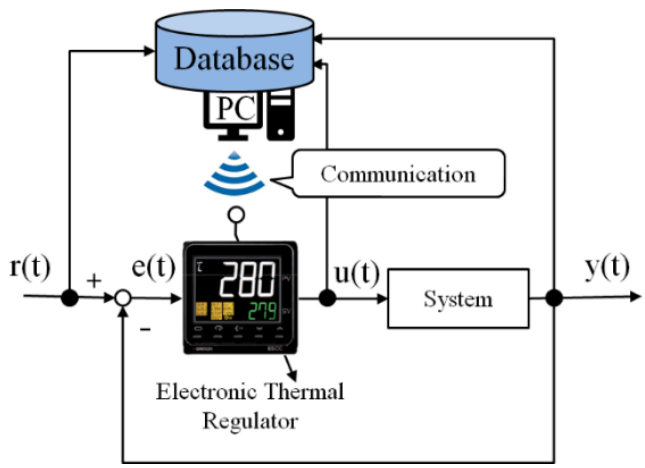

Fig. 1. The schematic of a data-driven control platform.

showing the historical data of the system. - 1) is defined by the following equation:

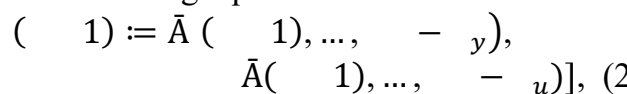

where is the control input, and ${ }_{y}, \overline{\mathrm{A}}_{u}$ are the orders of the system output and the control input, respectively. In this paper, the following control law with a PID structure is introduced:

$$
\begin{gathered}
()=\frac{c s}{I} \text { ( ) } \quad c \quad c \quad+\frac{d}{s}{ }^{2} \quad \text { ( ) } \\
I \text { ( ) } \quad P \quad \text { ( ) } D^{2}(), 3
\end{gathered}
$$

where ( ) denotes the control error signal defined by the following equation:

$$
\text { ( ) ( ) ( ), }
$$

where ( ) is the reference signal. The ${ }_{c},{ }_{i}$, and ${ }_{d}$ in equation (3) express the proportional gain, integral time, and derivative time, respectively. $P, I$ and $D$ similarly denote the proportional gain, integral gain and derivative gain. The ${ }^{-1}$ shows the backward operator. $s$ and $\left(:=1-^{-1}\right)$ are the sampling time and differencing operator.

\subsection{Design of a data-driven controller}

[STEP1] Generate an initial database: In the data-driven control, the control parameters are calculated from the historical closed-loop data stored in a database. If there are no stored information vectors in the database, the data-driven scheme cannot work. To create initial database, it is required to obtain the closed-loop data. The data stored in the form of the following equation:

$$
\begin{gathered}
(\mathrm{t}):=\left[^{-}(), \overline{\mathrm{A}}()\right], \overline{\mathrm{A}}=1,2, \cdots,(0) \\
():=\left[{ }_{P}(),{ }_{I}(),{ }_{D}()\right],
\end{gathered}
$$

where $(0)$ is the number of information vectors stored in the initial database. The query is defined by the following equation: 


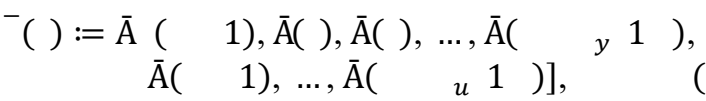

[STEP2] Calculate distances and Select neighbors: The distances between query ${ }^{-}()$and every information vector ${ }^{-}()$in the database, where, are calculated by the following equation:

$$
-(), \bar{A}())=\sum_{l=1}^{n_{u}+n_{y}+1}\left|\frac{-{ }_{l}()}{\max _{m}{ }_{l}\left({ }_{l}() \min _{m}{ }_{l}{ }_{l}()\right.}\right|,
$$

$$
(1,2, \cdots, \bar{A} \quad) \text { ) }
$$

where is the number of information vectors in the database at . The $\max ^{-}{ }_{l}()$ denotes the maximum value of th elements in all information vectors. Similarly, the $\min { }_{l}()$ expresses the minimum value of $t h$ elements in all information vectors. The $k$-pieces vectors with the smallest are selected from all information vectors. The selected information vectors and PID gains corresponding to the information vectors are neighbor vectors.

[STEP3] Calculate PID gains: The suitable PID gains around the query are calculated by applying the steepest descent method to selected neighbor vectors in STEP 2.

$$
\operatorname{old}()=\sum_{i=1}^{k} i(), \quad{ }_{i=1}^{k}=1, \quad 9
$$

where $i$ in equation (9) is weight for () corresponding to the $t h$ information vector ${ }^{-}()$as follows:

$$
i=\frac{-d_{i}}{\sum_{i=1}^{k}-d_{i}},
$$

where ${ }_{i}$ expresses the distance calculated by equation (8) between the query and $t h$ information vector.

\subsection{Off-line learning by FRIT}

The fictitious reference iterative tuning (FRIT) ${ }^{6}$ is a technique calculating directly the control parameters from a pair of I/O data. The new PID gains new are obtained by updating the database stored PID gains old. The following steepest descent method is applied to modify the PID gains:

$$
\text { new } \quad \text { old } \frac{(1)}{:=\left[{ }_{P}, \overline{\mathrm{A}}_{l}, \overline{\mathrm{A}}_{D}\right],}
$$

where denotes the learning rates for each control parameters. The ( 1 ) expresses the error criterion as follows:

$$
\begin{aligned}
& \text { ( } 1):=\frac{1}{2}(1)^{2} \\
& \left(\begin{array}{ll}
1 & 1
\end{array}\right):=\overline{\mathrm{A}}_{0}(1) \quad \tilde{m}_{m}\left(\begin{array}{l}
1 \\
1
\end{array}\right) \text {. }
\end{aligned}
$$

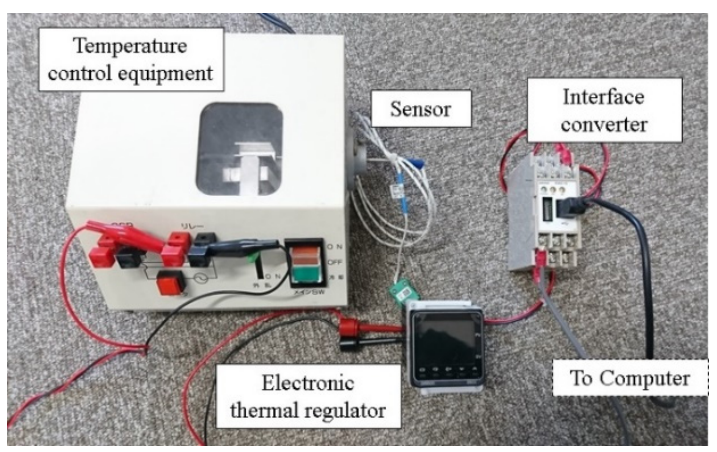

Fig. 2. The appearance of the temperature control equipment.

When ( 1 ) is minimized, the PID gains can be calculated such that 0() and $\tilde{m}_{m}()$ are equal. In equation (13), the fictitious reference output $\tilde{m}_{m}($ ) is used so that the closed-loop system including a controller has a desired characteristic of the reference model. ${ }_{m}($ ) is shown by the following equation:

$$
\tilde{m}_{m}()=\frac{{ }^{-1}(1)}{\left({ }^{-1}\right)} \sim() \text {. }
$$

$\left({ }^{-1}\right)$ is a user-specified polynomial included in the reference model and arbitrarily designed in advance such that the model has the desired characteristic.

$$
\begin{aligned}
& \left(^{-1}\right):=1+1^{-1} 2^{-2} \text {, } \\
& { }_{1}=-2 \exp \left(-\frac{\rho}{2 \mu}\right) \cos \left(\frac{\sqrt{4 \mu-1}}{2 \mu}\right. \\
& 2 \text { e } \operatorname{xp}\left(-\frac{\rho}{\mu}\right. \text { 、 } \\
& :=\frac{T_{s}}{\sigma} \\
& :=0.25(1 \bar{A} \bar{A})+0.51 \bar{A}
\end{aligned}
$$

denotes the rise-time, and is a parameter relevant to dumping. It is required that is set to between $1 / 2$ and $1 / 3$ sum of time constant and dead time. is desired to set to between 0 and 2 . When 0 , the step response is the Binomial model response. When 1 , the step response shows the Butterworth model response.

In equation (14), the fictitious reference input $\sim($ ) is calculated by the input-output relation of controller $\left({ }^{-1}\right)$. The $\left({ }^{-1}\right)$ is expressed as follows:

$$
\left(\begin{array}{lllll}
-1 & 0 & 1^{-1} & \ldots & n^{-n},
\end{array}\right.
$$

where is the control law order. equals 2 in the case where PID controller is used. The input-output relation of a controller is shown by the following equation:

$$
{ }_{0}()=\frac{\left({ }^{-1}\right)}{\Delta}\left\{\sim() \quad{ }_{0}()\right\},
$$

the fictitious reference signal $\sim()$ is obtained by deformation of equation (18). 


\section{Experimental control results}

In this paper, the data-driven control platform was applied to the temperature equipment shown in Fig. 2. The electronic thermal regulator E5CC-QQ2ASM and the interface converter K3SC-10 for connection between the electronic thermal regulator and a host computer was utilized, which they made by OMRON Corporation. The temperature equipment equips with a ceramic heater and an aluminum block. The control output ( ) is the temperature of the aluminum and is measured with a thermocouple of K-type. The control input ( ) is duty ratio which determines how much to heat through a relay. The reference signal $\overline{\mathrm{A}}(\mathrm{C})$ was set to ( ) $60\left[{ }^{\circ} \mathrm{C}\right]$ and the sampling time $s$ was set to 2 [s]. After the control output can track to the reference signal, a disturbance was applied that non-heated aluminum contacts and cools the heated one.

The PID parameters for generating initial database were denoted as follows:

$$
{ }_{P} 2 \text {.07, } \overline{\mathrm{A}}_{I}=0.021, \overline{\mathrm{A}}_{D}=1.29 \text {. }
$$

The initial data vectors were stored database according to equation (5). The user-specified parameters for the experiment are shown in Table 1.

The desired polynomial $\overline{\mathrm{A}}\left({ }^{-1}\right)$ was designed as

$$
\left({ }^{-1}\right)=1-1.91 \bar{A}^{-1} \quad 0 \quad .91^{-2} \text {, }
$$

where and were set to 90 and 0 . The control results of using the fixed PID gains and the proposed platform are respectively shown in Fig. 3. The upper figure shows that solid line is the control output by the proposed platform, dotted line is the desired control output, dash line is the reference signal and chain line is the control output using the fixed PID gains. The control result of using fixed PID controller cannot track to the desired control output and the disturbance let it cool $13^{\circ} \mathrm{C}$ approximately at $=700$. In comparison with two results, the rise-time of the proposed scheme is shorter than one of the fixed PID controller. Therefore, the effectiveness of data-driven control platform was verified.

\section{Conclusion}

In this paper, the effectiveness of the data-driven control platform has been verified. In the proposed platform, it is handily permitted advance control law that a host computer sends PID gains to the electronic thermal regulator using an existing interface. Even if PID gains cannot be sent to the electronic thermal regulator, a system is controlled using the last PID gains. In this paper, the PID gains are adjusted at every step. In future work, the control design method, in which PID gains are

\begin{tabular}{|c|c|}
\hline $\begin{array}{c}\text { Orders of the information } \\
\text { vector }\end{array}$ & $\begin{array}{ll}u & 1 \\
y & 2\end{array}$ \\
\hline Number of neighbors & 6 \\
\hline Learning rates & $\begin{array}{lll}P & 1 & .0 \times 10^{-3} \\
I & 1 & .0 \times 10^{-4} \\
D & 1 & .0 \times 10^{-3}\end{array}$ \\
\hline Initial Number of data & $(0)=1138$ \\
\hline
\end{tabular}

Table 1: User-specified parameters for the experiment.
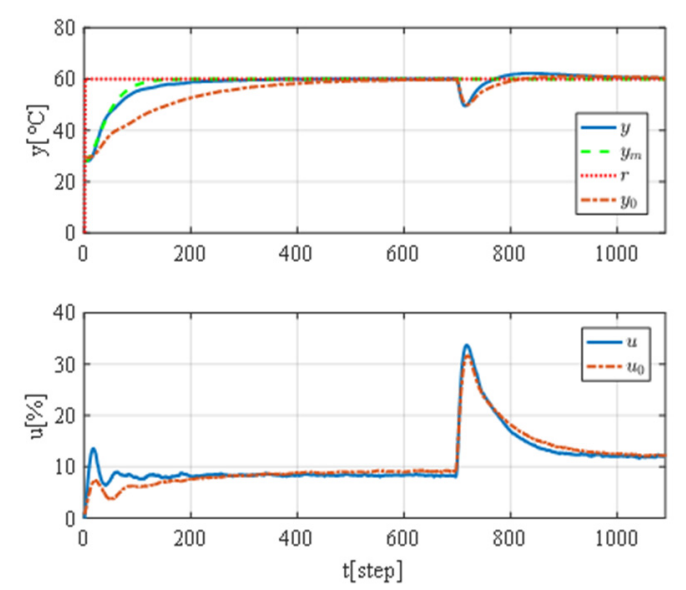

Fig. 3. The control results by the proposed platform, and by a fixed PID controller.

adjusted only the case where the control performance is deteriorated, will be considered.

\section{References}

1. T. Munaka, Newly Coming World by IoT-The Trend of Industrial Internet, System, Control and Information, Vol. 60, No. 10, 2016, pp. 425-430. (in Japanese)

2. Tateishi Electrric Co., Temperature Controller Thermac X Seriesss, Journal of The Society of Instrument and Control Engineers, Vol. 27, No. 12, 1988, pp. 1130-1131. (in Japanese)

3. K. J. Astrom, T, Hagglund, Advanced PID Control, International Society of Automation, (2005)

4. K.L. Chien, J.A. Hrones, and J.B. Reswick, On the Automatic Control of Generalized Passive Systems, Trans. ASME, Vol.74, 1972, pp. 175-185

5. T.Yamamoto, K.Takao, and T.Yamada, Desigh of a Data-Driven PID Controller, IEEE Transaction on Control Systems Technology, Vol.17, No.1, 2009, pp. 29-39

6. S. Soma, O. Kaneko, and T. Fujii, A New Approach to Parameter Tuning of Controllers by Using One-Shot Experimental Data: A Proposal of Fictitious Reference Iterative Tuning, Trans. Institute of Systems Control and Information Engineers, Vol.17, No.12, 2004, pp. 528-536 